\title{
FACTORIZATION AND INVARIANT SUBSPACES FOR NONCONTRACTIONS
}

\author{
BY JOSEPH A. BALL
}

Communicated by Mary Ellen Rudin, December 17, 1973

1. Introduction. The purpose of this note is to announce a generalization of the Sz.-Nagy-Foiaş model theory for contractions to arbitrary bounded operators. We also indicate how invariant subspaces are described by this model theory.

The Russians, for example, Livšic [14] and Brodskil̆ and Livšic [7], have studied model theories for various classes of operators, often including some noncontractions. Recently there has been some work, for example, Davis and Foiaş [13], Brodskiř, Gohberg, and KreǏn [9], and Brodskir [8] on characteristic functions for noncontractions. Our work is closely related to that of Clark [11] and depends heavily for inspiration upon the canonical models of de Branges-Rovnyak [5].

In many of these papers, one of the main points is the connection between factorizations of the characteristic function $B$ and invariant subspaces. Sz.-Nagy-Foiaş [15] found a precise condition on a factorization of $B$ to insure that it results (for contractions) in an invariant subspace. Also the work of de Branges [4], [5] should be mentioned. Most recently Clark [12] has taken this problem up for invertible noncontractions. We propose to study this problem for the class of bounded noncontractions.

2. Model theory. The characteristic operator function $B(z)$ of a bounded Hilbert space operator $T$ is defined by

$$
B(z)=-T J_{T}+z\left|I-T T^{*}\right|^{1 / 2}\left(I-z T^{*}\right)^{-1}\left|I-T^{*} T\right|^{1 / 2}
$$

where $J_{T}=\operatorname{sgn}\left(I-T^{*} T\right)$ and where $B$ acts from $\mathscr{D}_{T}$, the closure of the range of $\left|I-T^{*} T\right|^{1 / 2}$, to $\mathscr{D}_{T^{*}}$. A basic problem of model theory is to construct from $B$, in a canonical way, a bounded operator $T$ such that $B$ satisfies (1).

Let $\mathscr{C}_{*}$ and $\mathscr{C}$ be Hilbert spaces, and let $B(z): \mathscr{C} \rightarrow \mathscr{C}_{*}$ be analytic in a neighborhood $D$ of 0 . We also assume that $D$ is symmetric about the real line. Let

$$
J=\operatorname{sgn}\left(I-B(0)^{*} B(0)\right), \quad J_{*}=\operatorname{sgn}\left(I-B(0) B(0)^{*}\right), \quad \operatorname{sgn} 0=1 .
$$

AMS (MOS) subject classifications (1970). Primary 47A45. 
Let $\bar{B}(z)=B(\bar{z})^{*}$. For each $c \in \mathscr{C}_{*}, d \in \mathscr{C}$, let

$$
\begin{aligned}
& k(w, z)(c, d) \\
&=\left(\left[J_{*}-B(z) J B(w)^{*}\right] c /(1-z \bar{w})+[B(z)-B(\bar{w})] d /(z-\bar{w}),\right. \\
& {\left.[\bar{B}(z)-\bar{B}(\bar{w})] J c /(z-\bar{w})+\left[J-\bar{B}(z) J_{*} \bar{B}(w)^{*}\right] c /(1-z \bar{w})\right) ; }
\end{aligned}
$$

then $k(w, z)(c, d)$ is an ordered pair of functions each analytic in $z$ on $D$, the first component taking values in $\mathscr{C}_{*}$, the second in $\mathscr{C}$. Let $H_{0}=\left\{k(w, z)(c, d) \mid w \in D, c \in \mathscr{C}_{*}, d \in \mathscr{C}\right\}$ and $H_{1}$ be all linear combinations of elements of $H_{0}$. For $k\left(w_{1}, z\right)\left(c_{1}, d_{1}\right)$ and $k\left(w_{2}, z\right)\left(c_{2}, d_{2}\right)$ two elements of $H_{0}$, define

$$
\left\langle k\left(w_{1}, z\right)\left(c_{1}, d_{2}\right), k\left(w_{2}, z\right)\left(c_{2}, d_{2}\right)\right\rangle=\left\langle k\left(w_{1}, w_{2}\right)\left(c_{1}, d_{1}\right),\left(c_{2}, d_{2}\right)\right\rangle,
$$

the second inner product taken in $\mathscr{C}_{*} \times \mathscr{C}$, the elements of which are written as row vectors. Extend (3) to $H_{1}$ by linearity. It can be shown (see [1]) that a necessary condition that $B(z)$ be a characteristic function is that (3) be positive-definite.

When this is the case, we let $\mathscr{D}(B)$ (following notation of de BrangesRovnyak) be the completion of the pre-Hilbert space $H_{1}$. The elements of $\mathscr{D}(B)$ can be taken to be of the form $(f(z), g(z))$, where both $f$ and $g$ are analytic on $D, f$ is valued in $C_{*}, g$ in $\mathscr{C}$. When convenient, we write $(f, g)$ rather than $(f(z), g(z))$.

Define a linear operator $S: H_{0} \rightarrow H_{1}$ by

$$
\begin{aligned}
& S: k(w, z)(c, d) \rightarrow \\
& \quad \bar{w}^{-1}[k(w, z)(c, 0)-k(0, z)(c, 0)]+\bar{w} k(w, z)(0, d)-k(0, z)\left(J_{*} B(\bar{w}) d, 0\right)
\end{aligned}
$$

and extend $S$ to $H_{1}$ by linearity. The following theorem (proved in [1]) shows that this construction yields a model for a general bounded operator.

THEOREM 1. The operator $S$ extends by continuity to a bounded operator $($ also $S)$ on $\mathscr{D}(B)$, and $B$ coincides with the characteristic function of $S$. $A$ formula for $S$ independent of a kernel function representation is

$$
\begin{aligned}
S:(f(z), g(z)) & \rightarrow(z f(z)-B(z) J g(0),[g(z)-g(0)] / z) \text { and } \\
S^{*}:(f(z), g(z)) & \rightarrow\left([f(z)-f(0)] / z, z g(z)-\bar{B}(z) J_{*} f(0)\right) .
\end{aligned}
$$

Also proved in $[\mathbf{1}]$ are the useful relations

$$
I-S S^{*}=e_{1}(0)^{*} J_{*} e_{1}(0), \quad I-S{ }^{*} S=e_{2}(0){ }^{*} J e_{2}(0),
$$

where $e_{1}(0): \mathscr{D}(B) \rightarrow \mathscr{C}_{*}$ is defined by $(f, g) \rightarrow f(0)$ and $e_{2}(0): \mathscr{D}(B) \rightarrow \mathscr{C}$ is given by $(f, g) \rightarrow g(0)$. It follows from the definition of $\mathscr{D}(B)$ that

$$
e_{1}(0)^{*} c=k(0, z)(c, 0) \text { and } e_{2}(0)^{*} d=k(0, z)(0, d) .
$$


A consequence of Theorem 1 is that the positive-definiteness of the bilinear form (3) is necessary and sufficient for $B$ to be a characteristic operator function. There results a new proof of the theorem of Brodskil [8]. Clark [11] and Brodskiĭ, Gohberg and Kreĭn [9] handle the case where $B(z)$ is invertible in $D$.

3. Invariant subspaces. We restrict ourselves, for the purpose of studying invariant subspaces, to factorizations we call standard.

DEFINITION 1. For $B(z): \mathscr{C}_{1} \rightarrow \mathscr{C}_{3}$ a characteristic operator function, the factorization $B=B_{2} \cdot B_{1}\left(B_{1}(z): \mathscr{C}_{1} \rightarrow \mathscr{C}_{2}, B_{2}(z): \mathscr{C}_{2} \rightarrow \mathscr{C}_{3}\right)$ is said to be standard if:

(i) $B_{2}$ and $B_{1}$ are also characteristic operator functions.

(ii) On $\mathscr{C}_{1}, J_{1} \equiv \operatorname{sgn} I-B_{1}(0) * B_{1}(0)=\operatorname{sgn} I-B(0) * B(0)$.

On $\mathscr{C}_{2}, J_{2} \equiv \operatorname{sgn} I-B_{2}(0) * B_{2}(0)=\operatorname{sgn} I-B_{1}(0) B_{1}(0)^{*}$.

On $\mathscr{C}_{3}, J_{3} \equiv \operatorname{sgn} I-B_{2}(0) B_{2}(0)^{*}=\operatorname{sgn} I-B(0) B(0)^{*}$.

Let $[c, d]_{\imath}=\left\langle J_{\imath} c, d\right\rangle$ be the associated indefinite inner product on $\mathscr{C}_{i}$. Call an operator $X: \mathscr{C}_{i} \rightarrow \mathscr{C}_{k} J$-contractive if $[X c, X c]_{k} \leqq[c, c]_{\imath}, i, k=1,2$, 3. Then the condition for a factorization to be standard is essentially that $B(z)$ be the product of $J$-contractions. This generalizes the situation in the contraction case, where representations of $B$ as a product of contractions is studied (Sz.-Nagy-Foiaş [15]).

If $B(z)$ is of the form (1), we show that standard factorizations correspond to invariant subspaces, if not for $T$, then for $T \oplus U$, where $U$ is a unitary operator.

THEOREM 2. Let $B=B_{2} \cdot B_{1}$ be a standard factorization. Then there is a partial isometry $\Gamma$ from $\mathscr{D}\left(B_{2}\right) \oplus \mathscr{D}\left(B_{1}\right)$ onto $\mathscr{D}(B)$ given by

$$
\Gamma:\left(f_{2}, g_{2}\right) \oplus\left(f_{1}, g_{1}\right) \rightarrow\left(f_{2}+B_{2} f_{1}, \bar{B}_{1} g_{2}+g_{1}\right) .
$$

The difficulty for invariant subspaces is that $\Gamma$ may have a nontrivial kernel. The situation is best understood by defining another space, the overlapping space of de Branges and Rovnyak.

Definition 2. Let $B=B_{2} \cdot B_{1}$ be a standard factorization. Define a space $\mathscr{E}\left(=\mathscr{E}\left(B_{2} \cdot B_{1}\right)\right)$ by

$$
\mathscr{E}=\left\{(f, g) \mid\left(B_{2} f,-J_{2} g\right) \in \mathscr{D}\left(B_{2}\right) \text { and }\left(f,-\bar{B}_{1} J_{2} g\right) \in \mathscr{D}\left(B_{1}\right)\right\}
$$

with a norm given by

$$
\|(f, g)\|_{\mathscr{E}}^{2}=\left\|\left(B_{2} f,-J_{2} g\right)\right\|_{\mathscr{D}\left(B_{2}\right)}^{2}+\left\|\left(f_{1},-\bar{B}_{1} J_{1} g\right)\right\|_{\mathscr{D}\left(B_{1}\right)}^{2} .
$$

THEOREM 3. (i) $\mathscr{E}$ is isometrically isomorphic to $\mathscr{N}=$ the kernel of $\Gamma$ (see Theorem 2) under the map $\chi:(f, g) \rightarrow\left(B_{2} f,-J_{2} g\right) \oplus\left(-f, \bar{B}_{1} J_{2} g\right)$;

(ii) the operator $U$ defined by

$$
U:(f(z), g(z)) \rightarrow(z f(z)+g(0),[g(z)-g(0)] / z)
$$


is unitary on $\mathscr{E}$ with adjoint

$$
U^{*}:(f(z), g(z)) \rightarrow([f(z)-f(0)] / z, z g(z)+f(0)) .
$$

Note that (i) follows directly from the definitions. The proof of (ii) is a direct computation, using relations (4) and (5) in the appropriate spaces.

It follows from (ii) and Theorem 1 of de Branges [2] that $\mathscr{E}$ is a space of the type $\mathscr{E}(\varphi)$ studied by de Branges and Rovnyak [5].

The above analysis gives rise to an invariant subspace theorem, known to the Russians in terms of a somewhat different model theory [6].

THEOREM 4. Let $B=B_{2} \cdot B_{1}$ be a standard factorization. Let $S, S_{1}$ and $S_{2}$ be the model operators in $\mathscr{D}(B), \mathscr{D}\left(B_{1}\right)$ and $\mathscr{D}\left(B_{2}\right)$ respectively, and let $U$ be the unitary operator of Theorem 3 in $\mathscr{E}\left(B_{2} \cdot B_{1}\right)$. Then

$$
\begin{aligned}
\Gamma^{\prime} & =\left(f_{2}, g_{2}\right) \oplus\left(f_{1}, g_{1}\right) \\
& \rightarrow\left(f_{2}+B_{2} f_{1}, \bar{B}_{1} g_{2}+g_{1}\right) \oplus\left(\chi^{-1} P_{\mathscr{N}}\left(f_{2}, g_{2}\right) \oplus\left(f_{1}, g_{1}\right)\right)
\end{aligned}
$$

is unitary from $\mathscr{D}\left(B_{2}\right) \oplus \mathscr{D}\left(B_{1}\right)$ onto $\mathscr{D}(B) \oplus \mathscr{E}$, where $\mathscr{N}$ and $\chi$ are as in Theorem 3, (ii) $\mathscr{M}=\Gamma^{\prime}\left((0) \oplus \mathscr{D}\left(B_{1}\right)\right)$ is an invariant subspace for $S \oplus U$; $S \oplus U \mid \mathscr{M}$ is unitarily equivalent to $S_{1}$ via $\Gamma^{\prime}$, (iii) $\mathscr{M}^{\perp}=\Gamma^{\prime}\left(\mathscr{D}\left(B_{2}\right) \oplus(0)\right.$ ) is invariant for $S^{*} \oplus U^{*} ; S^{*} \oplus U^{*} \mid \mathscr{M}^{\perp}$ is unitarily equivalent to $S_{2}^{*}$ via $\Gamma^{\prime}$.

We hope to publish details elsewhere.

\section{REFERENCES}

1. J. A. Ball, Models for non-contractions (to appear).

2. L. de Branges, Factorization and invariant subspaces, J. Math. Anal. Appl. 29 (1970), 163-200. MR 40 \#7846.

3. - Some Hilbert spaces of analytic functions. II, J. Math. Anal. Appl. 11 (1965), 44-72. MR 35 \#778.

4. - Some Hilbert spaces of analytic functions. III, J. Math. Anal. Appl. 12 (1965), 149-186. MR 35 \#779.

5. L. de Branges and J. Rovnyak, Canonical models in quantum scattering theory, Perturbation Theory and its Applications in Quantum Mechanics (Proc. Adv. Sem. Math. Res. Center, U.S. Army, Theoret. Chem. Inst., Univ. of Wisconsin, Madison, Wis., 1965), Wiley, New York, 1966, pp. 295-392. MR 39 \#6109.

6. M. S. Brodskiľ, Triangular and Jordan representations of linear operators, "Nauka", Moscow, 1969; English transl., Transl. Math. Monographs, vol. 32, Amer. Math. Soc., Providence, R.I., 1971. MR 41 \#4283.

7. M. S. Brodskiĭ and M. S. Livšic, Spectral analysis of nonselfadjoint operators and intermediate systems, Uspehi Mat. Nauk 13 (1958), no. 1 (79), 3-84; English transl., Amer. Math. Soc. Transl. (2) 13 (1960), 265-346. MR 20 \#7221; 22 \#3982.

8. V. M. Brodskiǐ, On operator nodes and their characteristic functions, Dokl. Akad. Nauk SSSR 198 (1971), 16-19=Soviet Math. Dokl. 12 (1971), 696-700. MR 46 \#2458.

9. V. M. Brodskiĭ, I. C. Gohberg and M. G. Kreĭn, On characteristic functions of invertible operators, Acta Sci. Math. (Szeged) 32 (1971), 141-164. 
10. V. M. Brodskil and Ja. S. Švarcman, On invariant subspaces of contractions, Dokl. Akad. Nauk SSSR 201 (1971), 519-522=Soviet Math. Dokl. 12 (1971), 16591663. MR 45 \#2515.

11. D. N. Clark, On models for noncontractions, Acta. Sci. Math. (Szeged) (to appear).

12. - On functional models for non-contraction operators, (to appear).

13. C. Davis and C. Foiaş, Operators with bounded characteristic function and their J-unitary dilation, Acta. Sci. Math. (Szeged) 32 (1971), 127-139.

14. M. S. Livšic, On a certain class of operators in a Hilbert space, Mat. Sb. 19 (61) (1946), 239-262; English transl., Amer. Math. Soc. Transl. (2) 13 (1960), 61-83. MR 8, 588; 22 \#3981a.

15. B. Sz.-Nagy and C. Foiaş, Analyse harmonique des opérateurs de l'espace de Hilbert, Masson, Paris; Akad. Kiadó, Budapest, 1967; English rev. Transl., NorthHolland, Amsterdam; American Elsevier, New York; Akad. Kiadó, Budapest, 1970. MR 37 \#778; 43 \#947.

Department of Mathematics, Virginia Polytechnic Institute and State UNIVERSITY, BLACKSBURG, VirginIA 24061 